\title{
La discusión sobre el aborto provocado en el Congreso Nacional Brasileño: el papel del movimiento de mujeres
}

\author{
The discussion on induced abortion in the Brazilian \\ Congress: the role of the women's movement
}

Ellen Hardy 1

Ivanise Rebello 2

\footnotetext{
1 Departamento de Tocoginecologia, Faculdade de Ciências Médicas, Universidade Estadual de Campinas.

C. P. 6030 , Campinas, SP, 13081-970, Brasil.

2 Centro de Pesquisas das Doenças Materno-Infantis de Campinas.

C. P. 6181, Campinas, SP, 13081-970, Brasil.
}

Abstract Induced abortion has been discussed in Brazil for many years without producing changes in the Penal Code in force since 1940 and according to which it is illegal and a crime against life. There are only two circumstances in which induced abortion is allowed: when there is no other recourse for saving the pregnant woman' life, and when the pregnancy resulted from rapeand the abortion is preceded by the woman's informed consent. The purpose of this article is to show how the abortion problem has been dealt with under executive and legislative policies, focusing especially on discussion in Congress consi dering the role of organized women's groups. The study focuses on the legislative debate on induced aborti on and how it was preceded, accompanied, and followed by actions by the women's groups. Other political and social actors involved in the debate, such as the Catholic Church, sectors of the medical community, and protestant religions are mentioned.

Key words Abortion; Induced Abortion; Legislation; Women's Movement; Women's Health

Resumen El aborto provocado es un tema que ha sido muy discutido en Brasil desde hace mucho años, sin que se haya conseguido cambiar el texto del Código Penal vigente desde 1940. El Código considera que el aborto es ilegal y un crimen contra la vida. Solamente es permitido em dos casos: si no hay otra forma de salvar la vida de la embarazada o si el embarazo resulta de una violación y el aborto es precedido por el consentimiento dela embarazada. El objetivo de esteartículo es mostrar como se ha enfrentado el problema del aborto a nível de políticas gubernamentales y legislativas, especificamente la discusión en el Congreso Nacional considerando la actuación del movimiento de mujeres. En este ensayo es enfocado el debate sobre el aborto provocado en el poder legislativo y como éste fue precedido, acompañado y seguido por acciones del movimi ento organizado de mujeres. Otros actores políti cos y social es involucrados en el debate, tal como la I glesi a Católica, sectores de la categoria médica y las religi ones evangélicas, son mencionadas.

Palabras clave Aborto; Aborto Provocado; Legislación; Movimiento de Mujeres; Salud dela Mujer 
Introducción

La discusión sobre el aborto provocado movilizó recientemente a las delegaciones de todos los países presentes en la Conferencia Internacional de las Naciones Unidas sobre Población y Desarrollo realizada en el Cairo. El aborto provocado fue uno de los temas más controvertidos, exigiendo habilidad de los negociadores para llegar a un consenso, que fue el siguiente: "En ningún caso el aborto deberá ser promovido como un método de planificación familiar. Todos los gobiernos y organizaciones inter-gubernamentales e no-gubernamentales relevantes fueron Ilamados a intensificar su compromiso con la salud de las mujeres, a manejar el impacto del aborto inseguro como un gran problema de salud pública y a reducir la busqueda del aborto a través de la expansión y creación de servicios de planificación familiar. Siempre se dará a la prevención de embarazos no deseados la mayor prioridad y todos los intentos se harán para eliminar la necesidad del aborto. Las mujeres que tienen embarazos no deseados deberán tener facilidad de acceso a información confiable y a una consejería comprensiva. Todas las medidas o cambios en relación al aborto en el sistema de salud sólo pueden ser determinados a nivel nacional o local conforme el proceso legislativo del país. En las circunstancias en las cuales el aborto no sea contrario a la ley, estos deberán ser seguros. En todos los casos las mujeres deberán tener acceso a servicios de calidad para el manejo de las complicaciones resultantes del aborto. Consejería pós-aborto, educación y servicios de planificación familiar deberán ser ofrecidos prontamente, lo que también ajudará a evitar abortos repetidos" (ONU, 1994). La intensidad de los debates señaló la importancia del tema como un asunto que afecta a la población mundial.

Esto muestra que la discusión sobre el aborto provocado no puede ser abordada de manera simplista, por eso hacemos nuestras las palabras de la jurista Silvia Pimentel (1991) "En sana conciencia nadie es a favor del aborto. El aborto no es un bien en si mismo". Estamos frente a una discusión académica y también ante un gran problema social y de salud pública.

Una característica propia de este tema es que moviliza la emoción, principalmente de los que no entienden que al defender su legalización no se está preconizando su apología, sino que sea un derecho de la mujer que enfrenta un embarazo no planificado o de un feto malformado, incompatible con la vida.
El aborto provocado es la interrupción del embarazo como resultado de una intervención premeditada de la mujer o de terceros. En muchos países, incluyendo Brasil, el aborto es ilegal y considerado un crimen. El Código Penal brasileño considera que el aborto es un crimen contra la vida (una subclase de los crímenes contra la persona). Su texto dice lo siguiente:

Aborto provocado por la embarazada o con su consentimiento:

- Artículo 124 - provocar aborto en si misma o consentir que otro lo provoque. Detención de uno a tres años.

Aborto provocado por terceros:

- Artículo 125 - provocar aborto sin el consentimiento de la embarazada. Reclusión de tres a diez años.

- Artículo 126 - provocar aborto con el consentimiento de la embarazada. Reclusión de uno a cuatro años.

- Parágrafo único - se aplica la pena del artículo 126 si la embarazada no es mayor de 14 años, es enajenada o débil mental, o si el consentimiento se obtuvo mediante fraude, amenaza grave o violencia.

Forma calificada:

- Artículo 127 - las penas de los artículos 125 y 126 aumentan en un tercio si como consecuencia del aborto o de los medios para provocarlo, la mujer sufre un daño corporal grave, y son duplicadas si muere.

- Artículo 128 - No es punible el aborto practicado por médico: I - si no hay otra forma de salvar la vida de la embarazada. II - si el embarazo resulta de una violación y el aborto es precedido por el consentimiento de la embarazada, o cuando incapaz, de su representante legal (Código Penal Brasileiro, 1940).

El Código Penal fue promulgado en 1940 durante el "Estado Novo", una dictadura civil, dirigida desde 1937 por Getulio Vargas. Durante todo ese período el Congreso Nacional estuvo en receso, volviendo a la normalidad sólo en 1946. La parte general del Código fue modificada en 1984, pero hasta ahora no ha sido modificada la parte especial, donde se encuentran los artículos que penalizan el aborto (Pimentel, 1991).

Sin embargo, el hecho de ser un crimen y de que el Código Penal determine castigos para quienes lo practican no evita el problema. Su prohibición solamente obliga a las mujeres a hacerlo en la clandestinidad, en condiciones precarias de higiene, colocando su salud y su vida en riesgo.

En Brasil no existen estadísticas oficiales sobre el número de abortos practicados ni sobre el número de muertes resultantes. EI Mi- 
nisterio de Salud estima que son provocados 1,2 millón de abortos por año (Costa, 1993) y un estudio del Alan Guttmacher Institute (1994) concluyó que sería 1,4 millón por año. A pesar de la falta de informaciones confiables, el aborto provocado es uno de los problemas más complicados de salud pública en el país, porque no sólo tiene consecuencias médicas sino que también involucra factores éticos, sociales y religiosos.

El aborto provocado es un tema en el cual las autoras han trabajado hace algún tiempo, motivadas principal mente por la tragedia que significa para una mujer interrumpir un embarazo y también por la necesidad de informaciones concretas sobre las condiciones en que se hacen los abortos, las características de las mujeres que abortan y de como se sienten después. Además, les ha interesado la opinión que las mujeres tienen sobre el aborto y la legislación brasileña al respecto (Hardy et al., 1991; Hardy \& Alves, 1992; Osis et al., 1994).

Sin embargo, no es sólo sobre estos aspectos que se necesitan informaciones más fidedignas. El aborto provocado se viene discutiendo en el país hace cinco decadas y es interesante estudiar la evolución del debate. El objetivo de este trabajo es mostrar como se ha enfrentado el problema del aborto a nivel de políticas gubernamentales y legislativas, específicamente la discusión en el Congreso Nacional a la luz de la actuación del movimiento de mujeres.

Trás de cada proyecto de ley presentado al Congreso desde 1946, para cambiar el Código Penal en relación al aborto, han existido diferentes ideologías y actores políticos y sociales que participan además de influenciar en el debate. Estos son: Ios parlamentarios, el movimiento feminista, la I glesia Católica, sectores de la categoría médica y, en los últimos años, las religiones evangélicas.

El punto de vista feminista ha tenido un papel cada vez más importante en las acciones que dan visibilidad al tema del aborto, con su lucha por la concientización de la opinión pública. Muchas mujeres feministas se han comprometido directamente con algunas propuestas de modificar el Código Penal (Kosovsky et al., 1991; Portella, 1991).

En este ensayo pretendemos enfocar el debate sobre el aborto provocado en el poder legislativo en Brasil y como éste fue precedido, acompañado y seguido por acciones del movimiento organizado de mujeres. Los demás actores sociales involucrados en el debate serán mencionados, pero no se profundizará el análisis de su actuación.

\section{El debate sobre aborto en el Congreso} Nacional

El aborto provocado es un tema que ha ocupado un lugar importante en el país desde hace muchos años, sin haber conseguido cambiar el texto del Código Penal. Las propuestas y discusiones, para modificar la parte especial del Código donde se trata de los crímenes y de las penas, que han existido en diferentes sectores de la sociedad, incluyendo el Congreso Nacional y las Asambleas Legislativas Estaduales, fueron investigadas por diferentes autores. Se han presentado numerosos proyectos de ley, que proponían alteraciones específicas del Código Penal (Barsted, 1992; CFEMEA, 1993; Rocha, 1994). Siempre hubo propuestas para la total discriminalización, retirando los artículos correspondientes del Código Penal, y también propuestas de modificaciones graduales para ampliar las circunstancias en las cuales el aborto sería legal.

Rocha (1994) estudió detalladamente el debate sobre aborto en el Congreso, identificando cuatro etapas que se distinguen por el momento político en que ocurrieron, por un aumento gradual en el número de actores sociales involucrados y por el diferente número de legislaturas y propuestas.

En la primera etapa (seis legislaturas), que se extendió desde 1946 hasta 1971, se presentaron tres proyectos. El único favorable a la discriminalización (1965) argumentaba que el aborto provocado era un derecho de la mujer. De inmediato la I glesia Católica hizo pública su oposición. En ese período las mujeres brasileñas todavía no actuaban de forma significativa en esta área.

En la segunda etapa (tres legislaturas), de 1971 a 1983, gran parte de los proyectos todavia eran contrarios a la discriminalización o legalización del aborto. Casi todos los proyectos favorables daban una nueva redacción al artículo 128 del Código Penal, incluyendo el aborto terapéutico o necesario (practicado cuando la vida de la madre corre peligro). Los proyectos contemplaban el aborto, en el caso de psicosis grave y de debilidad mental. Además se incluía el aborto eugénico, definido como la interrupción del embarazo cuando se sospecha que el feto presenta graves anomalías o enfermedades transmitidas por uno o por los dos progenitores. Muchos de estos proyectos fueron influenciados por la categoría médica. En este período aún era pequeña la posibilidad de intervención organizada de las mujeres en el debate.

La tercera etapa (dos legislaturas) de 1983 a 1991, se caracterizó por la intensificación de la 
discusión sobre el tema, por un aumento importante en la cantidad de proyectos presentados, observándose un equilibrio entre el número de los contrarios y de los favorables a la discriminalización y/o a la legalización del aborto. La mayoría de los proyectos contrarios eran de parlamentarios conservadores, identificados con principios religiosos o con la categoría médica más tradicional. Los proyectos favorables eran de políticos de izquierda, influenciados por el movimiento de mujeres y el proceso de democratización del país. Además, en esa época ya habían algunas parlamentarias feministas.

En realidad, desde el comienzo de la decada del 80, con el proceso de re-democratización del país y la importante influencia del movimiento de mujeres, que inició un diálogo abierto con los partidos políticos y con las candidatas a cargo públicos, el debate se desarrolló con mayor intensidad. Esto se observó en las actividades ordinarias del Congreso, en las declaraciones de los parlamentarios, en el contenido de los proyectos de ley y también en los debates públicos.

Durante 1987 y 1988 se discutió la nueva Constitución (promulgada en Octubre de 1988) siendo el aborto provocado un tema muy controvertido. Uno de los puntos más discutidos fue la introducción o no, en el texto de la Carta Magna de la idea del "principio de defensa de la vida desde la concepción" que después de mucha polémica acabó no siendo incluída (Portella, 1991). Esto ha permitido continuar, hasta hoy, el debate para la modificación del Código Penal.

Durante los tres años transcurridos de la cuarta etapa (1991 a 1993) en la que ha habido una sola legislatura, se observó un gran cambio en las propuestas. Casi todas fueron favorables a la posibilidad de interrumpir el embarazo en otras circunstancias, además de las ya permitidas. Sin embargo, los dos proyectos de esta etapa contrários a la ampliación, fueron archivados definitivamente.

Se observa por lo tanto, que el tema ha llegado a un punto de intensas discusiones en el Congreso Nacional, donde la tendencia es favorable en general, pero hasta ahora no se ha conseguido alterar la legislación. Esta viene de una época cuando todavía no se conocía bien el funcionamento del aparato reproductivo femenino, ni los efectos y daños físicos y psicológicos resultantes de la práctica clandestina del aborto (Santos, 1993). Tampoco existían los exámenes pre-natales y procedimientos de diagnóstico intra-uterino actuales.
El movimiento de mujeres y el aborto

Al analizar el papel del movimiento de mujeres con relación al aborto provocado, es necesario señalar que ha habido un conflicto con relación al uso de dos palabras al discutir los posibles cambios del Código Penal: discriminalización y legalización. La discriminalización significa absolver un crimen, quitar la culpa, inocentar. Legalización significa hacer legal, auténtico, legítimo, justificado. De modo general, al movimiento de mujeres no le interesa sólo que el aborto deje de ser un crimen, quiere que sea normatizado y reglamentado, para que las mujeres que decidan hacerse un aborto puedan tener a su alcance servicios de salud accesibles y apropiados. Sin embargo, para concentrar esfuerzos, el movimiento cambió su estrategia, dando énfasis a la lucha por la discriminalización gradual. Se movilizó para que se cumpla la legislación vigente, com la creación de servicios de salud que hagan los abortos considerados legales, hasta que se consiga ampliar o cambiar el Código Penal. Esta posición está de acuerdo con la progresiva discusión y posterior liberalización de las leyes sobre aborto en numerosos países durante las dos últimas décadas. En todos ellos la tendencia ha sido retirar el enfoque criminal del aborto y resaltar la salud de la mujer y el bienestar de la familia (Cook, 1991).

Los principales años de movilización del Movimiento Feminista Brasileño contemporáneo fueron de 1979 a 1990, haciendo pública la discusión sobre el aborto, que hasta entonces estaba restringida a algunos estudios académicos (Milanesi, 1970; Fal coni, 1970) y a algunas organizaciones de mujeres (Alvarez, 1990). Estos grupos, formados por mujeres de todos los estratos sociales y políticos, se multiplicaron durante el período en cuestión.

En esos años algunos grupos comenzaron a centrar sus intereses en nuevos temas, que incluían anticoncepción, violencia contra la mujer, divorcio, planificación familiar y aborto. Como consecuencia se produjo un conflicto directo con la I glesia Católica, que hasta entonces había sido una fuerte aliada en la lucha contra la represión de la dictadura militar, y con algunos sectores del propio movimiento feminista (Alvarez, 1990; Barsted, 1992). Por otra parte, el ala izquierda del movimiento consideraba que la lucha por el aborto era una preocupación sólo de las burguesas e intelectuales.

En 1980, fueron detenidos, en una clínica en Río de Janeiro, pacientes, médicos y enfermeras acusados de hacer abortos. Esto produjo 
una reacción de algunas feministas que organizaron una manifestación pública frente a la Comisaría y al edificio de los Tribunales. Este hecho llevó a una campaña nacional para la discriminalización del aborto, durante la cual fue publicado el folleto “Mujeres Llegó la Hora de Luchar por el Aborto Libre", que era distribuído semanalmente en las ferias libres. Con esto las feministas llevaron el tema del aborto a las calles y a la prensa.

Se observó una actitud más ofensiva del movimiento feminista con relación al aborto durante el Primer Congreso de Mujeres Paulistas realizado em 1981. En esa oportunidad se hicieron acusaciones contra médicos sádicos, de un hospital universitario, que realizaban raspados sin anestesia, si empre que sospechaban que una mujer con hemorragia se había provocado un aborto. Muchas mujeres dijeron, y dicen, que no tienen derecho a escoger y que frente a un embarazo no deseado la única alternativa es un aborto clandestino. También en 1981, Jacqueline Pitanguy publicó en el "Jornal do Brasil" un artículo sobre el derecho de la mujer a optar por un aborto, recibiendo una fuerte reacción, en la prensa, de parte de la I glesia Católica que amenazaba con excomulgar a las que defendían el aborto (Barsted, 1992).

Durante 1983 ocurrieron una serie de acontecimientos relacionados con la lucha por la legalización del aborto. La campaña, ya mencionada, aumentó con el "Foro Feminista de Debates" en el cual el aborto apareció como parte del derecho a una sexualidad consciente. Como resultado de la campaña se publicó el boletin “Sexo Finalmente Explícito", que discutía temas de sexualidad, salud y aborto, y se escogió el día 28 de Setiembre como el Día Nacional de Lucha por la Legalización del Aborto. También en 1983, Martha Suplicy escribió un libro en que el aborto fue discutido sin preconceptos (Suplicy, 1983). Sorprendentemente, el libro fue publicado por una editora católica.

En este momento, el movimiento de mujeres alcanzó una gran fuerza en la sociedad pasando a ocupar espacio destacado en los medios de comunicación y en las esferas del poder público. En realidad, desdeel final de la década del 70 hubo un aumento en el número y en la importancia de los grupos de mujeres, asociado a las transformaciones sociales y económicas de la época. Es necesario recordar que a partir de 1977/ 78 aumentó la crisis de la legitimidad del gobierno militar, llegando a una gran movilización política y abriendo espacios para la actuación sindical y comunitaria. Esto hizo proliferar los movimientos sociales, con gran participación de mujeres, que paulatinamente fueron diferenciando (aborto y divorcio por ejemplo) sus reinvidicaciones específicas de la lucha más amplia por la redemocratización del país. Entre esas reinvidicaciones, se destacó la lucha por una mejor atención a la salud de la mujer, con gran énfasis sobre los derechos reproductivos (Osis, 1994).

La actuación de las mujeres en el área de la salud encontró respaldo en ideas, que venían siendo discutidas en el medio médico, acerca de una asistencia integral a la salud de la mujer. En una coyuntura política delicada como la descrita, las reinvidicaciones del movimiento de mujeres se sumaron a otras presiones y como consecuencia de ello, en 1983, el Gobierno Brasileño adoptó el Programa de Asistencia Integral a la Salud de la Mujer (PAISM). El Programa incluye el concepto de derechos reproductivos, que fue incorporado a la Constitución Federal (1988) y a varias Constituciones Estaduales (1989). Entre los objetivos del PAISM estaba evitar el aborto provocado, mediante la prevención del embarazo no deseado.

En 1984, se organizó un plebiscito informal, en Río de Janeiro, sobre la legislación punitiva relacionada con el aborto; $86 \%$ de las personas entrevistadas declararon ser contrarias a considerar el aborto como un crimen. En 1985, fue creado el Consejo Nacional de los Derechos de la Mujer (CNDM) con sede en el Ministerio de Justicia, al que convergieron las revindicaciones de los grupos organizados de mujeres. Desde el comienzo el CNDM reunió mujeres con diferentes ideologías y objetivos, que sin embargo, consiguieron disminuir tensiones políticas y divergencias (Alvarez, 1990). Los distintos grupos, que tenían en común un interés por la salud de las mujeres, señalaban la necesidad de que el Estado se hiciera cargo de las interrupciones legales de embarazos. Esto debería ser parte del PAISM, que incluiría ofrecer informaciones y servicios sobre anticonceptivos. Se presentaron dos proyectos para reglamentar la atención de los abortos legales en la red pública. El primero fue presentado en la Asamblea Legislativa del Estado de São Paulo por la Diputada Ruth Escobar, pero fue rechazado. La otra propuesta fue de la Diputada Lúcia Arruda que fue aprobada por la Asamblea Legislativa Estadual de Rio de Janeiro, pero revocada por el Gobernador, después de una intensa campaña contraria realizada por la Iglesia Católica.

A fines de 1988, el CNDM convocó a una reunión de abogados, en la Sección de Río de Janeiro de la Orden de los Abogados del Brasil (OAB), con el objetivo de promover los estudios necesários para adaptar la legislación 
brasileña ordinaria, incluyendo el Código Penal, a los dictámenes de la nueva Constitución. En 1989, el CNDM organizó en Brasilia el “Encuentro Nacional de Salud de la Mujer - Un Derecho a Ser Conquistado" que fue realizado en las dependencias del Congreso Nacional. Hubo una discusión específica sobre el aborto, donde fue contemplado desde la óptica de la ética, la política, la ley y la medicina. Durante este Encuentro se escribió la "Carta de las Mujeres en Defensa de su Derecho a la Salud" que en relación al aborto voluntario dice, entre otras cosas, que es un problema de la salud de la mujer, que los artículos que penalizan el aborto deben ser revocados porque la Constitución determina que la salud es un derecho de todos y un deber del Estado, y que éste debe atender los casos de aborto en la red pública de servicios de salud (federales, estaduales y municipales).

Después de este Encuentro el movimiento de mujeres continuó presionando a los políticos durante la preparación de las constituciones estaduales (1989) y las leyes orgánicas municipales (1990) para que fueran implantados servicios públicos para atender los casos de aborto permitidos por ley. El movimiento de mujeres siguió apoyando los proyectos favorables a la discriminalización y/ o legalización del aborto en el Congreso Nacional y participando en las discusiones de una propuesta para alterar el Código Penal.

En la década de los 90 continuaron las actividades para conseguir el derecho al aborto (Barsted, 1992; Santos, 1993) pero poco después del cambio de los dirigentes del CNDM en 1991, este órgano fue extinguindo de sus funciones. Como consecuencia el movimiento de mujeres perdió una parte importante de su poder de presión en el Congreso y en la sociedad, y hubo una disminución de la intensidad del proceso de lucha por el aborto provocado en el país. La crítica de la esterilización femenina indiscriminada y de las nuevas tecnologías reprodutivas pasaron a ser temas de mayor importancia. Esto se observa también en el contexto internacional, respaldado por la caída de los paradigmas políticos y por el crecimiento del fundamentalismo.

Si por un lado, encontramos todas estas discusiones y actividades en la sociedad, por otro poco se sabe sobre la opinión que la población femenina, la verdadera interesada, tiene sobre el aborto provocado en general, sobre la ley que lo rige y sobre cuales serían las modificaciones deseadas por ella. Excepto por unos pocos estudios (Ramos, 1983; Hardy, 1991) las voces de las mujeres casi nunca se oyen. En una investigación realizada en el Estado de São Paulo, se estudió la frecuencia del aborto provocado y las condiciones en que era hecho, además de la opinión de las entrevistadas sobre las circunstancias en que los hospitales deberían hacer abortos. En general las entrevistadas fueron más favorables a los casos previstos por la ley brasileña - estupro y riesgo de vida de la mujer - además de los casos de malformación fetal grave (Osis et al., 1994).

\section{Consideraciones finales}

El aborto provocado es un fenómeno mundial, que se observa en todas las civilizaciones. Referencias históricas muestran que ha sido practicado desde tiempos remotos. Sin embargo, nunca ha habido consenso sobre el tema. Por ejemplo, el aborto provocado fue condenado por la Iglesia Católica solamente a partir de 1869 (Muraro, 1989; 1993).

Hemos hecho más referencia a la Iglesia Católica porque Brasil es considerado un país de católicos. Todos los que luchan por la discriminalización/legalización del aborto quieren que el Estado asuma una posición Iaica y desvincule el aborto de la religión. Observamos, sin embargo, que aunque la I gl esia está separada del Estado desde la Proclamación de la República (1889), tiene el poder de influir y muchas veces de definir la posición del Estado, principalmente en los asuntos relacionados con moralidad y sexualidad (Barroso, 1991; Barsted, 1992; Rebello \& Hardy, 1992). La Iglesia ha mantenido siempre una postura dogmática de total criminalización del aborto.

En el Congreso Nacional las alas conservadoras de la I glesia actuan para frenar los proyectos liberales, en los bastidores de la política, a través de campañas intensas en la prensa y de "lobbies" poderosos. Por otra parte, han surgido grupos de legos y de religiosos que discuten el aborto en una posición no dogmática, reflexionando sobre las condiciones de vida de las mujeres y tomando una postura positiva frente a la legalización del aborto. Este es el caso de las Católicas por el Derecho de Decidir, una organización que apoya el derecho a la atención integral de la salud reproductiva, especialmente el acceso a métodos anticonceptivos y al aborto, en América Latina y en los Estados Unidos de América.

Un sistema como el brasileño, que no ofrece planificación familiar, a pesar de que en la Constitución consta que es deber del Estado educar al pueblo y ofrecerle atención médica y sanitaria, le está volviendo la espalda a gran 
parte de la población que recurre al aborto por absoluta falta de condiciones sociales, económicas y estructurales. Ni la religión católica, ni la legislación que castiga, afecta a las mujeres ricas que tienen recursos que les permiten hacerse un aborto en condiciones humanas. Las mujeres pobres son las realmente más perjudicadas.

Tal vez por eso es que personas que trabajan con poblaciones de pocos recursos muestran una posición mas comprensiva frente a la mujer que se provoca un aborto. Por ejemplo, la madre I vone Gebara, en entrevista dada a una revista de circulación nacional, declaró “Aborto no es pecado. El Evangelio ni lo menciona”. “... el dogma en relación al aborto fue fabricado a lo largo de los siglos". Otra de sus declaraciones fue contundente y refleja nuestro pensamiento: “El aborto es violento, muy violento. Siempre es una opción traumática, jamás un camino de alegría. La mujer sólo aborta si es obligada por las circunstancias. Sin embargo es una violencia que existe y como tal debe ser legislada. Frente al hecho de que el aborto es inevitable, es mejor hacerlo en condiciones dignas" (Nanne \& Bergamo, 1993). Varias personas reaccionaron a estas declaraciones, pidiendo que se preste atención a los crueles indicadores sociales del país (Pitanguy, 1993; Pinoti, 1994).

Recientemente se ha abierto un nuevo capítulo en la discusión sobre el aborto provocado. Conocidos profesionales del área de salud y jueces han hecho declaraciones, dándole nueva fuerza al debate, especialmente en la prensa. Tuvo gran repercusión a nivel nacional una entrevista hecha al Dr. Anibal Faúndes, Professor Titular de Obstetricia y Ginecología de una de las más prestigiadas universidades brasileñas, publicada en el diario de mayor circulación nacional. Este médico declaró públicamente que en su hospital se inducía el aborto en casos de fetos con diagnóstico de anencefalia (falta de cerebro) que no tenían posibilidad de sobrevivir (Camargo, 1994). Estas declaraciones Ilevaron a que se abriera una investigación criminal contra ese médico, por la práctica de aborto ilegal. Al mismo tiempo recibió amplio apoyo público, de instituciones tan importantes como la Sociedad Brasileña para el Progreso de la Ciencia y el Consejo Regional de Medicina de São Paulo.

Posteriormente, el presidente de la Sociedad Brasileña de Medicina Fetal, Dr. Thomas Gollop, declaró al mismo diario que también realizaba este procedimiento en casos de malformación fetal grave, cuando solicitado por la mujer (Camargo \& Feltrin, 1994).
La repercusión originada por la primera entrevista, tuvo la importancia de mostrar que la opinión pública estaba a favor de ampliar las condiciones en que el aborto debería ser legal. Por ejemplo, una encuesta de opinión realizada en São Paulo por la empresa Datafolha, reveló que $62 \%$ de la población consultada era favorable a la ampliación de la ley (Peres, 1994), y cuatro meses después de la publicación original, continuan los debates públicos sobre el asunto.

La discusión sobre el aborto en el Congreso Nacional, por los grupos de mujeres y en la sociedad en general, siempre tuvo un vaivén y los progresos alcanzados han sido mínimos. En este momento en que se está eligiendo un nuevo presidente además de senadores y diputados federales, la posición de los candidatos frente al aborto provocado es muy importante. No sólo porque la mayoría de la población brasileña es de sexo femenino (CFEMEA, 1993) sino también por que hay una Comisión responsable para proponer una reforma del Código Penal.

Al mismo tiempo, diversos jueces en varios Estados del Brasil, han autorizado judicialmente la interrupción del embarazo en casos de malformación incompatible con la vida (Correio Popular, 1994; Feltrin, 1994; Folha de São Paulo, 1994; Diário do Nordeste, 1994), creando jurisprudencia que refuerza la necesidad de reforma del Código Penal en lo que se refiere al aborto y demostrando que la lucha por la liberalización del aborto en Brasil no ha sido totalmente infructífera. 


\section{Referencias}

(THE) ALAN GUTTM ACHER INSTITUTE, 1994. Aborto Clandestino: Uma Realidade Latino-Americana. Nova York: The Alan Guttmacher Institute.

ALVAREZ, S. E., 1990. Engendering Democracy in Brazil. Women's movements in transition politics. New Jersey: Princeton University Press.

BARROSO, C., 1991. Consulta popular é oportuna. In: Leis e Políticas Sobre o Aborto - Desafios e Oportunidades. (S. Pimentel, org.), pp. 96-98, São Paulo: Conselho Estadual da Condição Feminina de São Paulo.

BARSTED, L. L., 1992. Legalização e descriminalização do aborto no Brasil: 10 anos de luta feminista. Estudos Feministas, 0:104-130.

CAM ARGO, L., 1994. Médico diz que U NICAM P faz aborto. Folha de São Paulo. São Paulo: 16 de junho. Caderno 3 - Cotidiano, p. 1.

CAMARGO, L. \& FELTRIN, R., 1994. Médico de SP afirma ter feito aborto ilegal. Fol ha de São Paulo. São Paulo: 24 de junho. Caderno 1 - p. 11.

CFEMEA (Centro Feminista de Estudos e Assessoria), 1993. Pensando Nossa Cidadania - Proposta para uma legislação não discriminatória. Brasília: CFEMEA.

CFEMEA (Centro Feminista de Estudos e Assessoria), 1993. Direitos da Mulher - O Que Pensam os ParIamentares. Brasília: CFEMEA.

CÓDIGO PENAL BRASILEIRO, 1940. Decreto-lei no 2848, 7 de dezembro.

COOK, R., 1991. Leis e Políticas Sobre o Aborto - Desafios e Oportunidades. São Paulo, Conselho Estadual da Condição Feminina de São Paulo.

CORREIO POPULAR, 1994. Juiz autoriza UNICAMP a fazer aborto de feto com má formação. Campinas: 13 de julho. Cidades, p. 3.

COSTA, A., 1993. Direitos Reprodutivos: riscos e encruzilhadas. Brasília. (mimeo.)

DIÁRIO DO NORDESTE, 1994. Decisão Inédita em Fortal eza. Justiça Autoriza Aborto de Feto Formado Sem Cérebro. Fortaleza: 26 de julho.

FALCONI, F. R. M., 1970. Incidencia de Aborto Provocado en el Amparo Maternal Durante el Año de 1967. Tese de mestrado, São Paulo: Faculdade de Higiene e Saúde Pública, Universidade de São Paulo.

FELTRIN, R., 1994. Justiça autoriza aborto em feto malformado. Folha de São Paulo. 12 de julho. Caderno 1 - Cotidiano, p. 9.

FOLHA DE SÃO PAULO, 1994. Advogada ganha direito ao aborto. São Paulo: 19 de julho. Caderno 4 Polêmica do aborto, p. 5.

HARDY, E.; REBELLO, I.; RODRIGUES, T. \& MORAES, T. M., 1991. Aborto provocado: diferenças entre pensamento e ação. Revista de Ginecologia eObstetrícia, 2:111-116.

HARDY, E. \& ALVES, G., 1992. Complicações pósaborto provocado: Fatores associados. Cadernos de Saúde Pública, 8:454-458.

KOSOVSKY, E.; ELUF, L. \& PIMENTEL, S., 1991. Proposta de alteração do Código Penal Brasileiro. In: Leis e políticas sobre o Aborto - Desafios e Oportunidades. (S. Pimentel, org.), pp. 111-116, São Paulo: Conselho Estadual da Condição Feminina de São Paulo.
MILANESI, M. L., 1970. O Aborto Provocado. São Paulo: Livraria Pioneira/ Editora Universidade de São Paulo.

MURARO, R. M., 1989. El aborto y la fe religiosa en América Latina. In: Mujeres e Iglesia - Sexualidad y Aborto en América Latina (A. M. Portugal, ed.) Washington: Publicación de Catholics for a Free Choice, M éxico/ Distribuciones Fontamara, S.A

MURARO, R. M., 1993. Uma história das idéias sobre o aborto. Folha deSão Paulo, 23 de novembro.

ONU (Organização da Nações Unidas), 1994. Programme of Action of the United Nations International Conference on Population and Development. Cairo: ONU.

OSIS, M. J. D., 1994. Atenção Integral à Saúde da MuIher, o Conceito e o Programa. História de uma Intervenção. Tese de Mestrado, Campinas: Departamento de Antropologia, Instituto de Filosofia e Ciências Humanas, Universidade Estadual de Campinas.

OSIS, M. J. D.; HARDY, E.; FAÚNDES, A.; ALVES, G. \& BALAREZO, G., 1994. Opinião das mulheres sobre as circunstâncias em que os hospitais deveriam fazer abortos. Cadernos de Saúde Pública, 10: 320-330.

PERES, A., 1994. Aborto: a defesa sai da clandestinidade. Claúdia: São Paulo: outubro.

PIMENTEL, S., 1991. Apresentação. In: Leis e Políticas Sobre o Aborto - Desafios e Oportunidades. (S. Pimentel, org.), pp. 7-8, São Paulo: Conselho Estadual da Condição Feminina de São Paulo.

PIMENTEL, S., 1991. Aborto: alguns dados e considerações jurídicas. Seminário Nacional "A Realidade do Aborto no Brasil", Coletivo Feminista Sexualidade e Saúde, Estudos e Comunicação em Sexualidade e Reprodução Humana, e Instituto da MuIher Negra, Embu, São Paulo. (mimeo.)

PINOTI, A. J., 1994. Legalização do aborto, uma triste necessidade. Fol ha de São Paulo, São Paulo: 11 de setembro.

PITANGUY, J., 1993. Chega de bancar o avestruz. Veja, São Paulo: 25 de agosto.

PORTELLA, A. P., 1991. Aborto: uma abordagem da conjuntura nacional e internacional. Seminário Nacional "A Realidade do Aborto no Brasil", Coletivo Feminista Sexualidade e Saúde, Estudos e Comunicação em Sexualidade e Reprodução Humana, e Instituto da Mulher Negra, Embu, São Paulo. (mimeo.)

REBELLO, I. \& HARDY, E., 1992. Sexo não desejado, violência, desigualdade de gênero e o papel dos grupos de mulheres. Relatório do Workshop, São Paulo. (mimeo.)

ROCHA, M. I. B., 1994. O Congresso Nacional ea questão do aborto. Relatório Preliminar de Pesquisa, Campinas: Núcleo de Estudos de População, Universidade Estadual de Campinas. (mimeo.)

SANTOS, C. A., 1993. Aborto: Um Olhar Displicente da Sociedade. Tese de Mestrado, Belo Horizonte: Centro de Desenvolvimento e Planejamento Regional, Faculdade de Ciências Econômicas, Universidade Federal de Minas Gerais.

SUPLICY, M., 1983. Conversando Sobre Sexo. São Paulo: Editora Vozes. 